\title{
77. Shifts in the Spectral Lines of Ionised Titanium by Disruptive Discharge.
}

By Hantaro Nagaoka, Tetsugoro Futagami and Iwajiro Machida.

Institute of Physical and Chemical Research.

(Rec. July 8, 1930. Comm. by H. NaGaOKA, M.I.A., July 12, 1930.)

The spectrum of singly ionised titanium was already studied by Russell." The result is somewhat at variance from our experiment, by which the lines from $5000 \AA$ down to $2500 \AA$ were studied. The method of observation was the same as that given for the iron spectrum. ${ }^{2)}$ Specially to be remarked are the differences in the reversal of some lines; what Russell describes as unsymmetrically reversed is due to greater red shading (Type II), while the case of greater violet shading (Type III) is generally classified as symmetric reversal. Among the nonreversed series of Russell, one or two of the lines at the head of the series show faint reversal, which is mostly symmetrical. This discrepancy seems to rest on the strength of the disruptive discharge. The term notations here used are those of Russell.

I. (Fig. 1) In this type the lines are displaced towards the red with slight broadening, which is to be recognized in some series. Generally the head line is most affected. The series showing this type of shift are given below, with the number of lines examined $\mathrm{N}$, and the displacement of the head line $\mathrm{J} \nu$, measured from the middle of the displaced position. Sometimes faint reversal can be traced in a number of lines.

\begin{tabular}{|c|c|c|c|c|}
\hline Series & $\mathrm{N}$ & $h, k$ & $\lambda$ & $-\Delta v$ \\
\hline$a^{2} D_{h}-a^{2} D_{k}^{\prime}$ & 4 & 3,3 & 4294.10 & 0.75 \\
\hline$a^{4} P_{h}^{\prime}-a^{4} D_{k}^{\prime}$ & 8 & 3,4 & 4300.05 & 0.7 \\
\hline$b^{2} F_{h}^{\prime}-c^{2} D_{k}^{\prime}$ & 2 & 4,3 & 4163.65 & 0.7 \\
\hline$a^{2} P_{h}^{\prime}-a^{4} D_{l i}^{\prime}$ & 3 & 2,3 & 4399.77 & 0.7 \\
\hline$a^{4} P_{h}^{\prime}-a^{2} D_{k}^{\prime}$ & 2 & 2,3 & 4417.71 & \\
\hline$a^{2} H_{l}^{\prime}-a^{2} G_{l i}^{\prime}$ & 3 & 6,5 & 4549.64 & \\
\hline$a^{2} G_{h}-a^{2} F_{h}$ & 3 & 5,4 & 4468.49 & \\
\hline$a^{2} P_{h}^{\prime}-a^{2} D_{k}^{\prime}$ & 2 & 2,3 & 4533.97 & \\
\hline$c^{2} D_{b}-c^{2} F_{l b}$ & 1 & 3,4 & 4488.32 & \\
\hline$a^{2} D_{h}-a^{2} F_{b}$ & 3 & 2,3 & 4443.80 & \\
\hline
\end{tabular}

1) Russell: Astrophys. Journ. 66 (1930), 87.

2) Nagaoka, Futagami a. Machida: Proc. 6 (1930), 146, 224. 
II. (Fig. 2) The lines are somewhat unsymmetrically reversed, the shading being greater towards the red. The series showing this type of shift are the following:-

\begin{tabular}{lccccc}
\multicolumn{1}{c}{ Series } & $\mathrm{N}$ & $h, k$ & $\lambda$ & $-\Delta y$ & $+\Delta y$ \\
$b^{4} F_{h}^{\prime}-a^{4} F_{h}$ & 8 & 5,5 & 3322.93 & 7.24 & 6.34 \\
$a^{2} D_{h}-b^{2} F_{l s}$ & 2 & 3,4 & 3190.87 & 5.39 & 4.92 \\
$b^{4} F_{h}^{\prime}-a^{4} D_{h}^{\prime}$ & 7 & 5,4 & 3168.52 & 5.48 & 3.58 \\
$a^{2} H_{h}^{\prime}-b^{2} G_{k}^{\prime}$ & 2 & 6,5 & 3224.23 & 4.81 & 4.34 \\
$b^{2} D_{h}-b^{2} D_{h}^{\prime}$ & 2 & 3,3 & 3741.33 & 4.50 & 3.21 \\
$a^{4} P_{h}^{\prime}-a^{4} P_{k}$ & 3 & 3,3 & 3058.08 & 3.21 & 1.81
\end{tabular}

III. (Fig. 3) The reversal is slightly asymmetric, the red shading being smaller than the violet. A large number of lines belong to this type. The heads of the series show distinct reversal, but in lines of higher terms, it becomes somewhat obscure.

$\begin{array}{lrrrrr}\text { Series } & \mathrm{N} & h, k & \lambda & -\Delta y & +\Delta y \\ a^{2} F_{h}^{\prime}-a^{2} D_{k}^{\prime} & 2 & 4,3 & 3685.19 & 10.06 & 11.20 \\ a^{4} F_{h}^{\prime}-a^{4} G_{k}^{\prime} & 6 & 4,5 & 3361.22 & 7.86 & 9.36 \\ a^{4} F_{h}^{\prime}-a^{4} D_{k}^{\prime} & 7 & 5,4 & 3088.03 & 6.32 & 8.54 \\ a^{4} F_{h}^{\prime}-a^{4} F_{k} & 10 & 5,5 & 3234.52 & 7.65 & 8.13 \\ a^{2} F_{h}^{\prime}-a^{2} G_{k}^{\prime} & 2 & 4,5 & 3349.02 & 5.72 & 7.16 \\ b^{4} P_{h}^{\prime}-b^{4} D_{k}^{\prime} & 2 & 3,4 & 3248.60 & 6.64 & 7.11 \\ b^{2} G_{h}-b^{2} G_{k}^{\prime} & 2 & 5,5 & 3504.89 & 5.29 & 5.70 \\ a^{2} F_{h}^{\prime}-a^{2} F_{k} & 3 & 4,4 & 3759.30 & 4.46 & 5.45 \\ b^{2} G_{h}-a^{2} H_{k} & 2 & 4,5 & 3287.64 & 4.16 & 4.91 \\ b^{4} P_{h}^{\prime}-a^{4} S_{k}^{\prime} & 1 & 3,2 & 3332.10 & 3.37 & 5.33 \\ b^{4} P_{h}^{\prime}-a^{4} P_{k} & 2 & 3,3 & 3106.23 & 3.50 & 4.03 \\ b^{2} G_{h}-c^{2} F_{k} & 1 & 5,4 & 3103.80 & 3.28 & 4.02 \\ a^{2} D_{h}-b^{2} D_{k}^{\prime} & 1 & 3,2 & 3278.91 & 3.32 & 3.98\end{array}$

IV. (Fig. 4) The lines are shifted towards the violet and broadened without shading. The mean shift is given by $\Delta \nu_{1}$, and the breadth by $\Delta \nu_{2}$. It is to be remarked that the breadth of the undisturbed lines lies between the limits $d \nu=0.2$ to 0.8 .

$\begin{array}{cccccc}\text { Series } & \mathrm{N} & h, k & \lambda & +\Delta v_{1} & \Delta v_{2} \\ a^{2} F_{h}^{\prime}-b^{2} D_{k}^{\prime} & 3 & 3,3 & 2868.73 & 2.03 & 9.8 \\ b^{2} P_{h}^{\prime}-c^{2} D_{k}^{\prime} & 2 & 2,3 & 3535.40 & 1.92 & 7.8 \\ a^{2} F_{h}^{\prime}-a^{4} F_{k}^{\prime} & 3 & 4,3 & 3836.10 & 0.82 & 1.4 \\ a^{4} F_{h}^{\prime}-a^{2} D_{k}^{\prime} & 5 & 3,3 & 3130.81 & 0.61 & 3.2 \\ a^{2} F_{h}^{\prime}-a^{4} D_{k}^{\prime} & 4 & 4,4 & 3587.13 & 0.54 & 2.3\end{array}$




$\begin{array}{llllll}b^{2} G_{h}-c^{2} F_{l_{k}} & 2 & 4,3 & 3089.39 & 0.52 & 4.7 \\ b^{2} G_{h}-b^{2} F_{l_{b}} & 2 & 5,4 & 4028.35 & 0.36 & 2.8 \\ a^{2} P_{h}^{\prime}-a^{2} S_{l_{k}}^{\prime} & 2 & 2,1 & 3641.33 & 0.36 & 6.8 \\ a^{2} F_{h}^{\prime}-a^{4} G_{k}^{\prime} & 2 & 3,3 & 4012.40 & 0.31 & -\end{array}$

V. The lines are not displaced, but mostly only broadened. There are a large number of lines belonging to this type.

\begin{tabular}{llccc}
\multicolumn{1}{c}{ Series } & $\mathrm{N}$ & $h, k$ & $\lambda$ & $\Delta \vee$ \\
$a^{2} F_{h}-c^{2} G_{k}$ & 2 & 4,5 & 2751.70 & 19.2 \\
$a^{4} G_{h}^{\prime}-a^{4} H_{k}^{\prime}$ & 6 & 6,7 & 2828.15 & 18.8 \\
$a^{2} G_{h}-b^{2} G_{k}^{\prime}$ & 4 & 5,5 & 2884.10 & 13.9 \\
$a^{2} G_{h}^{\prime}-b^{2} H_{k}^{\prime}$ & 2 & 5,6 & 2954.76 & 12.8 \\
$a^{4} F_{h}-a^{4} D_{k}$ & 6 & 5,4 & 2800.65 & 12.7 \\
$a^{4} F_{h}-a^{4} G_{k}$ & 6 & 5,6 & 2945.47 & 11.5 \\
$a^{2} F_{h}-d^{2} F_{k}^{\prime}$ & 3 & 3,3 & 2931.27 & 11.4 \\
$a^{4} G_{h}^{\prime}-a^{4} G_{k}$ & 8 & 6,6 & 2856.24 & 10.8 \\
$a^{2} G_{h}^{\prime}-c^{2} G_{k}$ & 2 & 5,5 & 3022.83 & 7.7 \\
$a^{2} F_{h}-c^{2} F_{k}^{\prime}$ & 2 & 4,4 & 3128.64 & 7.4 \\
$b^{2} P_{h}^{\prime}-b^{2} P_{k}$ & 3 & 2,2 & 3456.40 & 4.1 \\
$a^{2} P_{h}^{\prime}-a^{2} P_{k}$ & 3 & 2,2 & 3374.34 & - \\
$b^{2} D_{h}-b^{2} F_{k}$ & 2 & 3,4 & 3659.75 & - \\
$b^{2} D_{h}-a^{2} P_{k}$ & 3 & 3,2 & 3724.08 & -
\end{tabular}

The different types of shifts are shown in the diagram.

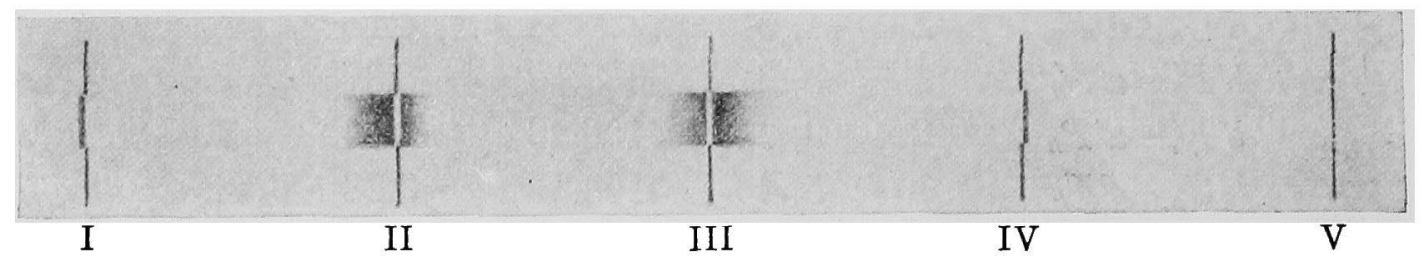

As will be clear from the figures, the ionized lines excited by disruptive discharge are very strong compared with those obtained in the arc.

Microphotometric studies of the lines for $\mathrm{Fe}, \mathrm{Ni}$ and $\mathrm{Ti}$ will be published in the Scientific Papers of the Institute. 


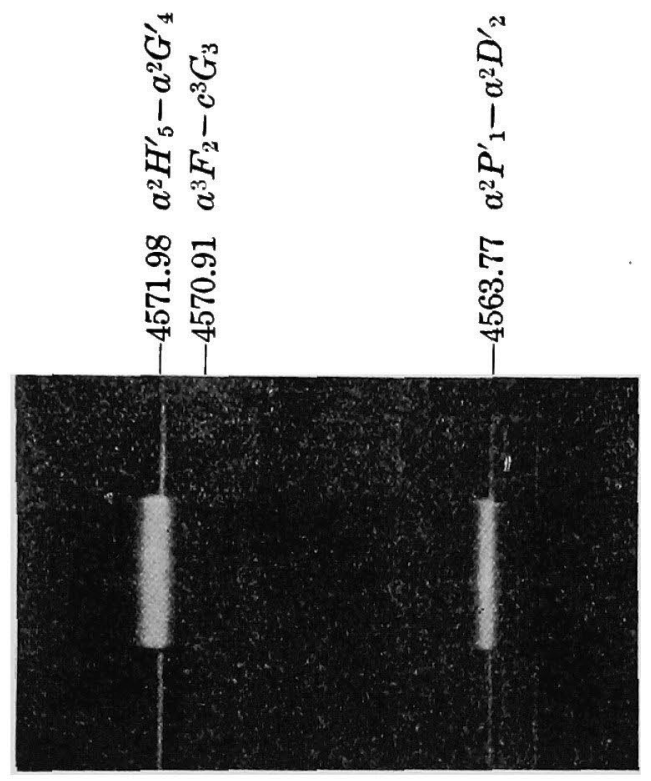

Fig. 1.

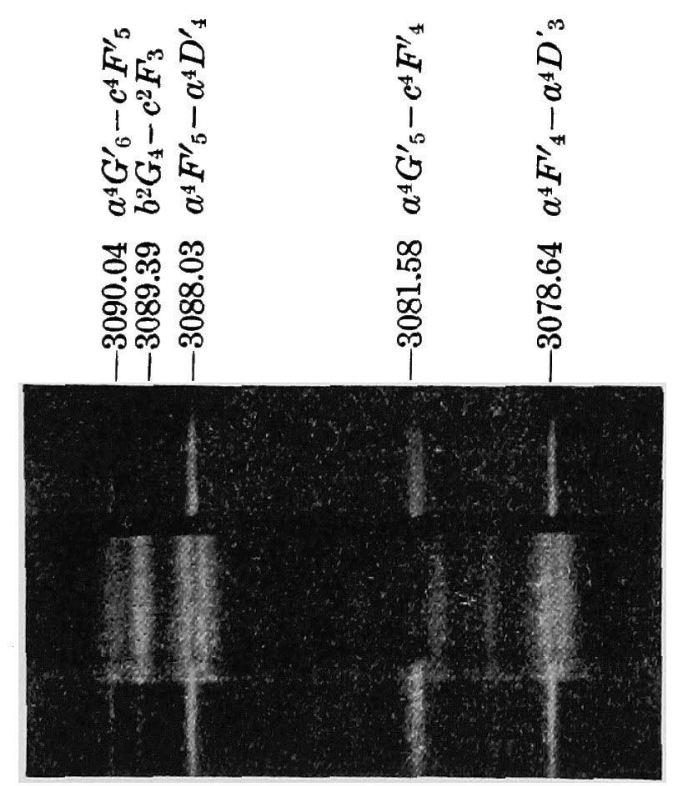

Fig. 3 .

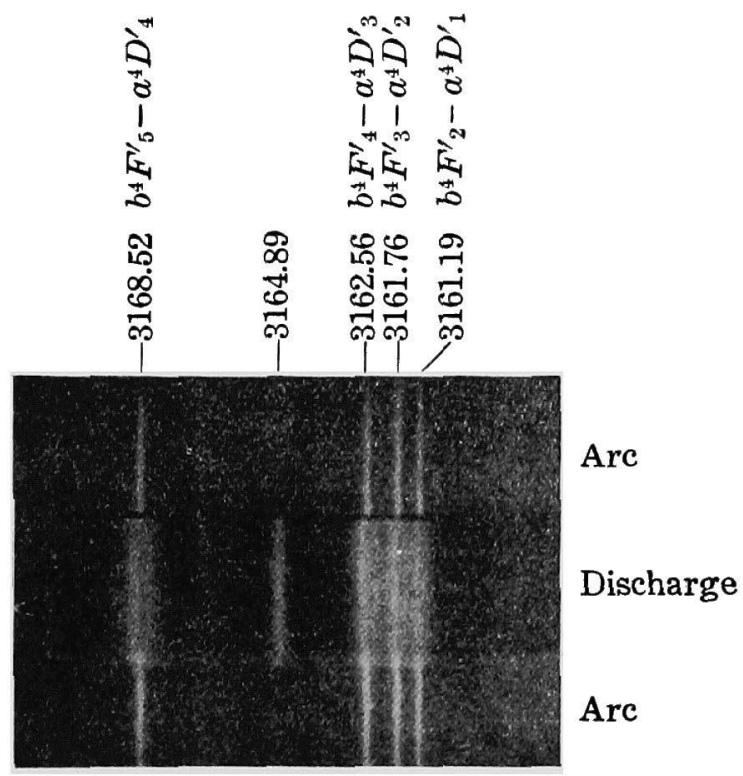

Fig. 2.

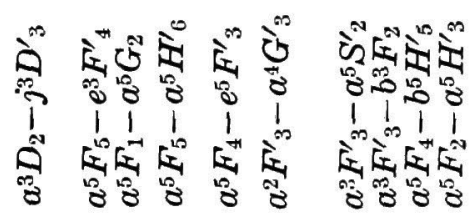

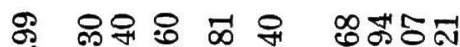

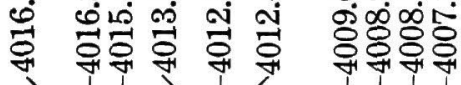

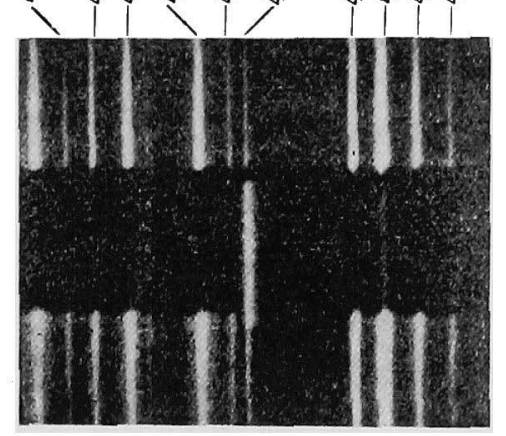

Arc

Discharge

Arc

Fig. 4. 